\title{
Usefulness of a Concept Called Autonomous Selection
}

\author{
Idan S. Solon \\ University of Central Florida, Burnett School of Biomedical Sciences \\ Orlando, Florida, United States 32816 \\ Correspondence: IdanSolon@gmail.com
}

Orcid ID: 0000-0002-1480-2552

\begin{abstract}
Here, I introduce a concept called autonomous selection to refer to a source of selection that is part of the individuals upon which it acts. The concept is motivated by a set of phenomena with the following characteristics: Natural selection shaped a variant (e.g., gene, epigenetic mark, or combination thereof) to act in a manner that reduces the frequency of one or more heritable traits of the individual in which it is located if those traits are detrimental to individual or group fitness. Phenomena with these characteristics are peculiar to traditional evolutionary theory but have been identified rather frequently in recent decades. They are also relevant to adaptive evolution: By reducing the frequency of a trait detrimental to fitness, the variant accelerates the evolution of adaptations, which allows its holders to adapt better to constantly changing environments. The variant is shaped by (natural) selection, but also does (autonomous) selection.
\end{abstract}

Several phenomena with these characteristics have been invoked by proponents of the extended evolutionary synthesis (EES). The concept of autonomous selection helps resolve some of the controversy surrounding the EES: EES proponents call attention to the incompleteness of contemporary theory, emphasizing individuals' processes that influence which adaptations those individuals evolve. I argue for the special importance of individuals' processes that do not just influence those individuals' adaptations, but also accelerate the adaptive evolution of those individuals. All known phenomena that fit this description are examples of autonomous selection. Other phenomena raised by EES proponents do not meet this threshold. 
Keywords: extended evolutionary synthesis; inheritance of acquired characters; stress-induced mutagenesis; fitnessdependent sex; horizontal gene transfer 


\section{Introduction}

Since Darwin (1859) introduced the metaphor of natural selection, researchers have discussed selection in many senses, including life history stages of selection (e.g., fecundity selection, viability selection, mortality selection); sources, or mechanisms, of selection (e.g., sexual selection, ecological selection; artificial selection, parental selection); and units, or targets, of selection (e.g., genic selection, individual selection, group selection).

Autonomous selection ${ }^{1}$ is introduced here to refer to a source of selection that is part of the individuals upon which it acts. The concept, autonomous selection, is motivated by a set of phenomena with the following characteristics: Natural selection shaped a variant (e.g., gene, epigenetic mark, or combination thereof) to act in a manner that reduces the frequency of one or more heritable traits exhibited by the individual in which it is located if those traits are detrimental to individual or group fitness. (I consider potential examples in sections 3 to 7 ). Phenomena with these characteristics are peculiar to traditional evolutionary theory but have been studied in recent decades.

By reducing the frequency of heritable traits that lower fitness, the variant (in this manuscript's examples) accelerates the evolution of adaptations compared to natural selection acting in the absence of the variant. This occurs similarly to how condition-dependent sexual selection accelerates the evolution of adaptations (Lorch et al. 2003). Accelerating the evolution of adaptations is tantamount to improving the adaptive fit of organisms in their environment because of the following. The relationship between organisms and environment is constantly in flux, as organisms continuously adapt to an ever-changing environment. These changes include the evolution of predators, parasites, and prey and changes in weather. Organisms are imperfectly adapted to their environment because of time-lags: It takes time for organisms to adapt to a new environment, at which point the environment has changed. Therefore, when a variant accelerates adaptive evolution, it also shortens the time-lag, so organisms become better-adapted. Because the environment constantly changes, the variant perpetually causes organisms holding it to be better-adapted to their environment. These examples are qualitatively different from examples in which processes alter adaptation but do not enhance it.

\section{Criteria for autonomous selection}

\footnotetext{
${ }^{1}$ Autonomous selection is not to be confused with autoselection, which refers to the evolution of genomic elements that replicate to the benefit of themselves, rather than a higher unit of selection (Bell 1997).
} 
Evolution by selection in any sense is characterized by: (1) a nonrandom ${ }^{2}$ change in the frequency ${ }^{3}$ of one or more traits that are (2) heritable.

It can be confusing to attempt to identify a "source of selection" - that is, a source responsible for a nonrandom change in the frequency of heritable traits - simply because of the challenges in precisely identifying a source responsible for any act. Indeed, any act is preceded by millions of years of antecedent causes. On what grounds is one singled out as responsible? Nevertheless, biologists have been willing to single out a source of selection, given an indication that that source "purposely" evaluates traits and favors some over others. Biologists have considered particular instances of human agency to act as sources of selection, for example, parental selection (Harris 2006), artificial selection (Darwin 1859), and volitional selection (Wilson 2014). In these examples, a sense of purpose is suggested by expressed human preference. However, more pertinently to this manuscript, this suggestion of purpose can also follow from an indication that the source has been shaped by natural selection to evaluate traits and favor some. For example, natural selection has shaped a female's choice among males on the basis of the traits they exhibit, justifying the designation sexual selection ${ }^{4}$ (Darwin 1871).

As a consequence of evolution by selection, a unit of selection becomes better-suited to a life history stage (e.g., in fecundity selection, viability selection, mortality selection) or a source (e.g., in sexual selection, artificial selection, parental selection) responsible for the nonrandom change in frequency of heritable traits, assuming a constancy of the life history stage or the source responsible for the change in trait frequency. If there is a source of selection responsible for the source of selection itself, this better-suitedness to the source of selection can be enabled by a better-suitedness to the original source. For example, female preferences for condition-dependent traits in males are under natural selection. The resulting sexual selection leads males to evolve to be better at maintaining their condition and, therefore, better at attracting females than they would be.

Selection has been discussed above as having two characteristics: (1) a nonrandom change in the frequency of one or more traits that are (2) heritable. Examples in this manuscript are considered to constitute autonomous selection if they check off these two criteria and a third, which is that natural selection has shaped a variant to cause

\footnotetext{
2 The terms "nonrandom," "average," and "consistent" are often used to distinguish selection from drift.

${ }^{3}$ This change in frequency has been variously called a "frequency...increase" (Brooker et al. 2014, pg. 7), "changes in proportions" (Futuyma 2009, pg. 8), and a "consistent difference in fitness" (Futuyma 2009, pg. 283).

${ }^{4}$ Sexual selection in other contexts corresponds more to life history stages of competition for reproductive success (Mayr 1997).
} 
a change that meets criteria 1 and 2 among traits of the same individual in which the variant is located. By doing so, the variant would be considered to do autonomous selection.

The examples of autonomous selection in this manuscript meet these three criteria by the following: A variant has been shaped by natural selection to act in a manner that reduces the frequency of heritable traits of the same individual if those traits reduce individual or group fitness. I discuss these three criteria further below. Then in sections 3 to 7 , I discuss particular findings and whether they satisfy the three criteria.

Criterion 1: Is there nonrandom change in the frequency of one or more traits?

The "change" considered here is in comparison to what would have been expected in the cause's absence, not in comparison to what occurred previously. This allows autonomous selection to encompass processes that accelerate adaptive evolution that fit the former characteristic but not the latter. For example, DNA repair and the piRNA silencing of transposons (section 6.3) satisfy criterion 1 under the former interpretation. These changes are nonrandom because they specifically target, respectively, recent errors and transposons.

The trait need not actually be exhibited: If there is bias against it, this is a change compared to what would occur otherwise; therefore, developmental bias (section 7.3) against certain combinations of traits satisfies criterion 1.

The "nonrandom" requirement means hypermutation (section 4) does not satisfy criterion 1: In hypermutation, mutations at a locus occur at an elevated rate irrespective of which alleles are at the locus.

A number of findings involve a decrease in the frequency of a trait if it is associated with stress: stressinduced mutagenesis (section 3); stress-induced genetic transfer (section 6.2); and fitness-dependent sex (section 5). If natural selection has shaped the changing of genes or genetic combinations in response to the stressor, it is because of the potential to generate genes or genetic combinations associated with more adaptive traits that would not be stress-afflicted and that, consequently, would not be changed. Therefore, any change induced as a result of stress-induced mutagenesis, horizontal gene transfer, or fitness-dependent sex is considered nonrandom, even if most, or all, of the individuals in the population at a particular time are subjected to the genetic changes.

Criterion 2: Is the change sufficiently heritable to constitute evolution? 
Evidently, natural selection shaped specific epigenetic modifications to occur in response to specific environmental stimuli (Dickins and Rahman 2012). Disputes over whether those modifications constitute Lamarckian evolution (Jablonka and Lamb 2008, 2010; Dickins and Barton 2012; Dickins and Rahman 2012) have been muddled by confusion about what the terms "proximate" and "ultimate" mean (Haig 2013). However, these debates have seemed to hinge upon whether these modifications are sufficiently stable across generations to constitute evolution. This discussion can also be extended to include examples of natural selection for specific genomic modifications that may be short-lived on an evolutionary timescale, such as CRISPR (section 6.1) and small viral DNA (section 6.4). Since the question is whether these modifications are "sufficiently" heritable, it is more likely to be decided by empirical advances than the other two criteria.

Criterion 3: Has natural selection shaped a variant to favor or disfavor one or more heritable traits? That is, are criteria 1 and 2 met because natural selection has shaped a variant to induce a change that meets these criteria?

It was discussed above that biologists have been willing to delineate a source of selection if natural selection has shaped that source. For example, natural selection has shaped female choice, which is called sexual selection. Therefore, the satisfaction of criterion 3 meets that threshold for delineating the category of autonomous selection.

However, criterion 3 has greater significance to the study of adaptive evolution. The examples of autonomous selection in this manuscript all meet criterion 3 via natural selection of a variant that disfavors (i.e., selects against) traits associated with lower fitness and thereby favors traits associated with higher fitness, thereby accelerating adaptive evolution and allowing organisms to adapt faster to an ever-changing environment.

Selfish genetic elements (Trivers and Burt 2006) do not satisfy criterion 3 because they are not shaped by natural selection to act conditionally depending upon indications about the phenotype of the individual in which they are located.

Criterion 3 is seldom (if at all) met by examples of niche construction (see section 7.1) and phenotypic plasticity (see section 7.2) that have been emphasized by EES proponents. Even if natural selection has shaped these processes and even if these processes change the organisms' relationship to their environment and consequently influence which traits those organisms evolve, this does not mean that natural selection shaped the processes for the purpose of favoring these traits. 


\section{Stress-induced mutagenesis}

Stress-induced mutagenesis (SIM) is an increase in mutation rates in response to stress and/or maladaptedness (Jablonka and Lamb 2005; Mittelman 2013). SIM has been documented in a broad range of species, including bacteria, yeast, algae, nematodes, flies, and humans (Al Mamun et al. 2012; Shee et al. 2012; Mittelman 2013; Ram and Hadany 2016). Elevated rates of many different kinds of mutations have been documented in response to many different kinds of stress (reviewed in Shapiro 2011). Rates of epigenetic changes also increase in response to stress (Shapiro 2011), though biologists have focused upon genetic mutations when arguing that natural selection shaped an increase in mutation rates in response to stress. That is, there is more evidence that SIM satisfies criterion 3. Therefore, it receives the focus in this section.

Criterion 1: Is there nonrandom change in the frequency of one or more traits?

By imposing a higher mutation rate in response to stress, SIM reduces the frequencies of traits more associated with stress compared to traits less associated with stress. This change in frequency is nonrandom because the traits associated with stress are likely to be less adaptive than other traits (Ponder et al. 2005).

Criterion 2: Is the change sufficiently heritable to constitute evolution?

SIM has often been studied in single-celled organisms (e.g., bacteria, yeast, algae) which pass mutations to offspring. Mutations generated via SIM in multi-cellular organisms can be passed to offspring as well (Jablonka and Lamb 2005, pg. 99).

Criterion 3: Has natural selection shaped a variant to favor or disfavor one or more heritable traits?

In recent decades, multiple theoretical and empirical analyses have concluded that stress-induced mutagenesis has been shaped by natural selection - that is, that natural selection has shaped an elevation in the mutation rates to occur in response to stress, whether these increases in mutation rates have occurred at specific genomic sites or regions or to a stressed individual globally (Jablonka and Lamb 2005; Ponder et al. 2005; Shee et al. 2011; MacLean et al. 2013; Ram and Hadany 2016). These biologists have argued that SIM has been selected in order to evolve traits that are better able to respond to the source of the stress (Galhardo et al. 2007; Shee et al. 2011). Theoretical evidence in favor of natural selection of SIM includes mathematical models demonstrating that SIM can gain frequency (Galhardo et al. 2007). Empirically, there is "overwhelming” evidence of natural selection of SIM, 
including but not limited to genomic analyses; the identification of mutation pathways; and the ability to use gene knockouts in order to prevent a mutation rate increase (Galhardo et al. 2007; Shee et al. 2011).

\section{Local hypermutation}

In local hypermutation (Jablonka and Lamb 2005; Hodgkinson and Eyre-Walker 2011; Martincorena et al. 2012; Martincorena and Luscombe 2012), mutation rates are higher at some loci than at others and this elevated mutation rate is thought to remain consistent from individual to individual and through time - that is, it is not "induced" by stressors (Jablonka and Lamb 2005, pg. 94). Locally-elevated mutation rates have been found in taxa ranging from bacteria to humans. Jablonka and Lamb (2005, pg. 92) consider local hypermutation to be a source of mutations that is "not entirely random, but...also not precisely directed." That is, more mutations are produced at a genomic place where they are useful, though the specific mutations that occur are determined randomly.

Insofar as a locally-elevated mutation rate is the same across the population, then they do not satisfy criterion 1 of autonomous selection. Local hypermutation may cause changes in the frequencies of traits, but these changes are random with respect to the properties of the traits themselves. That is, though there is nonrandomness with respect to which locus is subjected to mutations, there is no nonrandomness with respect to which alleles at a particular locus are subjected to mutations. Therefore, no alleles are made to lose or gain frequency on a nonrandom basis as a consequence of the elevated mutation rate. Criterion 1 is not met.

\section{Fitness-dependent sex}

In many species, sex is an obligate aspect of reproduction (Hadany and Beker 2007; Colegrave 2012). However, in some taxa (including bacteria, yeast, viruses, nematodes, crustaceans, clovers, and soil microfungi), sex is practiced facultatively and the frequency of sex depends upon the condition of the individuals: Stressed and/or less fit individuals undergo sexual reproduction at higher rates, while unstressed and/or more fit individuals are more likely to practice asexual reproduction (Hadany and Beker 2003; Hadany and Otto 2007; Michod 2011; Ram and Hadany 2016). Facultative sex can accelerate the evolution of adaptation (Hadany and Otto 2009).

Criterion 1: Is there nonrandom change in the frequency of one or more traits?

Compared to asexual reproduction, sexual reproduction breaks up genetic associations (Bell 1997; Agrawal 2006;

Otto 2009). Therefore, genetic associations that are subjected to sexual reproduction lose frequency. If organisms 
initiate sex on the condition that they are stressed or otherwise indicated to be less fit, then their genetic associations are subjected to sexual reproduction on a nonrandom basis.

Criterion 2: Is the change sufficiently heritable to constitute evolution?

Facultative sex is theorized to have an important evolutionary role, accelerating the evolution of adaptations (Ram and Hadany 2016).

Criterion 3: Has natural selection shaped a variant to favor or disfavor one or more heritable traits?

Biologists have offered theoretical and empirical support for theories that natural selection shaped stress-induced sex (Hadany and Beker 2003; Hadany and Otto 2007; Otto 2009; Ram and Hadany 2016). Multiple models (Hadany and Beker 2003; Hadany and Otto 2007) demonstrate that an allele can evolve by natural selection to cause its holder to have sexual reproduction if its fitness is low, but asexual reproduction if its fitness is high.

\section{Inheritance of acquired adaptive characters}

Biologists have been alert to the idea of inheritance of acquired characters since Lamarck and perhaps eons before (Zirkle 1935). This idea fell out of favor after the Darwinian revolution, but has gained momentum again in recent decades. Koonin (2019) outlined two criteria for "inheritance of acquired adaptive characters": (1) specific, heritable changes in the genome caused by an external factor and (2) a specific phenotypic effect of those changes that constitutes adaptation to the causative factor. Findings that satisfy Koonin's two criteria for the inheritance of acquired adaptive characters also constitute autonomous selection, provided that natural selection has shaped a mechanism in the inheriting organism's genome by which the organism inherits the acquired adaptive characters.

Koonin (2019, Table 1) has proposed multiple examples of phenomena that satisfy his two criteria, including CRISPR-Cas with strong self versus non-self discrimination; specific cases of horizontal gene transfer; piRNA defenses against transposable genetic elements; and virophage-mediated defense against giant viruses in protists. I discuss these events with respect to the three criteria for autonomous selection.

\subsection{CRISPR}

Bacteria and archaea cells use the CRISPR system to target genetic material that resembles spacer material in the cellular genome (Horvath and Barrangou 2010; Sorek et al. 2013; Hille et al. 2018). Shapiro (2011, pg. 79) called 
the CRISPR system "a precise example of the kind of dedicated, nonrandom, beneficial change specifically excluded by generalizations of evolutionary theorists." However, Weiss (2015) argued that CRISPR systems do not actually discriminate between genetic material that is valuable and that which is parasitic. Koonin and Wolf (2016) distinguished between CRISPR systems that incorporate random DNA segments to serve as spacers and CRISPR systems that incorporate DNA from viruses. The latter are biased toward targeting non-self DNA. Koonin and Wolf argued that there would be natural selection for the latter kind of CRISPR system (so, it satisfies criterion 3 ). Here, I focus on CRISPR-Cas systems with strong self versus non-self discrimination.

Criterion 1: Is there nonrandom change in the frequency of one or more traits?

The trait that applies to criterion 1 - the trait that is changed by the CRISPR system-is the genetic material targeted by the system. This targeted material is a cognate of the genetic spacer material held in the organism's genome. When the genetic spacer material changes, the system's target changes and this target change represents a trait change. Since there is a bias toward targeting non-self genetic material, the CRISPR system is responsible a nonrandom change.

Criterion 2: Is the change sufficiently heritable to constitute evolution?

The genetic material inside the spacers is responsible for what material is targeted by the CRISPR system. This genetic material is transmittable to subsequent generations. CRISPR-mediated heredity may be short-lived on an evolutionary timescale, but CRISPR inserts are sufficiently heritable to be considered Lamarckian according to Koonin and Wolf (2009).

Criterion 3: Has natural selection shaped a variant to favor or disfavor one or more heritable traits?

Mechanisms for self vs. non-self discrimination seem to occur in at least some CRISPR systems (Koonin 2018). Koonin and Wolf (2016) have argued that there is natural selection for CRISPR systems that exhibit strong non-self discrimination. Such CRISPR systems are better at defending the organism against an infection.

\subsection{Horizontal gene transfer}

Horizontal gene transfer (or lateral gene transfer) occurs when organisms acquire genetic material from organisms other than their parents (Ochman et al. 2000; Koonin et al. 2001; Keeling and Palmer 2008; Treangen and Rocha 
2011; Soucy et al. 2015). Horizontal gene transfer has been documented in both unicellular and multicellular organisms.

Criterion 1: Is there nonrandom change in the frequency of one or more traits?

Genetic material acquired by horizontal transfer can result in adaptations (Koonin et al. 2001; Kay et al. 2002; Gyles and Boerlin 2014). But this does not necessarily mean that there is a bias in horizontal gene transfer toward receiving genes associated with adaptiveness or toward replacing genes associated with maladaptiveness. Some biologists have argued that horizonal gene transfer is not Lamarckian because there is no bias toward transferring variants that are adaptive to the receiving individual (Weiss 2015; Roger 2018). Others have considered it a Lamarckian process (Vetsigian et al. 2006; Koonin and Wolf 2009; Martin 2018; Koonin 2019). There is some evidence that horizontal gene transfer occurs more by stressed organisms (Prudhomme et al. 2006; Stevens et al. 2011; Slager et al. 2014; Domenech et al. 2018), which satisfies criterion 1 because traits associated with maladaptiveness become more subjected to change.

Criterion 2: Is the change sufficiently heritable to constitute evolution?

Genes acquired by horizontal transfer can be inherited by offspring and play a significant evolutionary role (Ochman et al. 2000; Keeling and Palmer 2008; Polz et al. 2013; Gyles and Boerlin 2014).

Criterion 3: Has natural selection shaped a variant to favor or disfavor one or more heritable traits?

Theory holds that there can be natural selection of some significant rate of lateral gene transfer and empirically, the rate of lateral gene transfer can be high (Vos et al. 2015). Biologists generally accept that natural selection has shaped mechanisms by which organisms receive genetic material via horizontal transmission in order to allow organisms to adapt to environmental novelty (Koonin et al. 2001; Gogarten et al. 2002; Burrus and Waldor 2004; Gogarten and Townsend 2005; O’Malley and Boucher 2005; Nielsen et al. 2014; but see Vogan and Higgs 2011).

\section{3 piRNA defenses against transposable elements}

Transposable elements move around a genome and can harm (e.g., sterilize) the individuals in which they are located. Organisms employ piRNA to silence transposable elements in germ cells (Gross 2006; Aravin et al. 2007; Halic and Moazed 2009; Siomi H. and Siomi M. 2015; Charlesworth et al. 2017). In order to silence transposons, 
the piRNA system requires the DNA of previously active transposable elements to guide it. As multiple authors have noted, transposon silencing is similar to CRISPR, which requires the DNA of previously virulent viruses in order to determine which viruses to target (Shapiro 2011; Koonin 2019). Transposon silencing with piRNA has primarily been studied in Drosophila melanogaster.

Criterion 1: Is there nonrandom change in the frequency of one or more traits?

Biologists believe that the transposon silencing occurs to prevent maladaptive traits, including sterility (Aravin et al. 2007; Siomi H. and Siomi M. 2015; Toth et al. 2016; Parhad and Theurkauf 2019). The silencing, therefore, conducts a nonrandom influence upon an organism's traits.

Criterion 2: Is the change sufficiently heritable to constitute evolution?

Genetic clusters of previously active transposable elements are used to guide the piRNA system to target transposons. These genetic clusters are passed to offspring. The inheritance is, therefore, genetic. The transposon silencing is conducted epigenetically.

Criterion 3: Has natural selection shaped a variant to favor or disfavor one or more heritable traits?

piRNA machinery that facilitates transposon silencing appears to have been shaped by natural selection (Aravin et al. 2007; Parhad and Theurkauf 2019).

\subsection{Virophage defenses}

Fischer and Hackl (2016) documented a phenomenon by which a small virus protects a unicellular eukaryote from a large virus, as follows. The small virus is integrated into the eukaryote's genome and its genes are expressed when the cell is infected by the large virus (Fischer and Hackl 2016; Koonin and Krupovic 2016). The infection usually kills the cell but after the cell's death, the large virus and the small virus are released into the environment. The small virus evidently benefits neighboring cells by reducing the large virus's reproduction rate during attacks upon the neighbors.

Criterion 1: Is there nonrandom change in the frequency of one or more traits?

The trait that is changed when the cell incorporates a small virus into its genome and releases it into the environment is the specific small virus that is released into the environment after death, as opposed to another small virus that 
could have been released. The change is nonrandom because the small virus that is released is more likely to be one that is useful for neighbors.

Criterion 2: Is the change sufficiently heritable to constitute evolution?

The small viral DNA is included into the eukaryotic cell's genome, so it is potentially heritable enough to be considered Lamarckian (Koonin 2019).

Criterion 3: Has natural selection shaped a variant to favor or disfavor one or more heritable traits?

Biologists have surmised that natural selection for the virophage defense occurred on the kin level (Koonin and Krupovic 2016).

\section{The extended evolutionary synthesis}

Since the modern evolutionary synthesis in the $20^{\text {th }}$ century, some biologists have proposed novel syntheses due to what they have felt were inadequacies of prevailing evolutionary theory in accounting for certain empirical phenomena. For example, relatively recently, Arthur (2004) proposed an "inclusive synthesis" that would accommodate findings of developmental bias and Jablonka and Lamb (2005) discussed a "new synthesis" that would accommodate epigenetic, behavioral, and symbolic inheritance. However, it is Pigliucci's (2007) call for an "extended evolutionary synthesis" (EES) that has been most responsible for inspiring scholarly discussion about a novel synthesis. Over the ensuing decade, the EES has generated considerable attention, confusion, and controversy, the last two of which are at least partially due to a lack of consistent articulation of the EES's essence. Pigliucci (2007, pg. 2743) acknowledged that he was offering “a stimulus to much needed discussion, not a fullfledged outline of a new research program."

The EES has represented different claims to different biologists at different times. However, recent articles written by both skeptics and proponents of the EES suggest that the controversy centers on the role of organisms in the evolution of their own adaptations. For example, Laland et al. (2015, pg. 7) of the proponents wrote of a claim that lends the EES "considerable coherence across topics": "Developmental processes play important evolutionary roles as causes of novel, potentially beneficial, phenotypic variants, the differential fitness of those variants, and/or their inheritance (i.e. all three of Lewontin's conditions for evolution by natural selection.) Thus, the burden of creativity in evolution (i.e. the generation of adaptation) does not rest on selection alone." As a further illustration, 
Charlesworth et al. (2017, pg. 2) in the skeptical camp wrote that the single EES claim that "seriously" challenges the modern synthesis is the claim that "natural selection acting on 'random' mutations is inadequate to explain adaptive evolution."

For EES proponents, “organisms co-direct their own evolution” (Laland et al. 2014, pg. 162) through developmental processes that "instigate" (Laland et al. 2014, pg. 162), "modify" (Odling-Smee et al. 2003, pg. 16), and "bias" (Laland et al. 2015, pg. 7) selection. Here, I establish a distinction between two influences a developmental process can exert on the adaptive evolution of the organisms in which the process occurs. A process can: 1) lead to the evolution of different adaptations than otherwise or 2) not only lead to the evolution of different adaptations than otherwise, but also accelerate adaptive evolution. This first category is all-encompassing.

Virtually every developmental process that organisms undertake can be said to change the organisms' characteristics and relationship to their environment and, therefore, change how natural selection acts upon the organisms, which influences what adaptations evolve. The same can be said for any environmental change: Any environmental change can alter how natural selection acts upon organisms and influence what adaptations evolve. A minor alteration can produce substantial effects, per the butterfly effect. Therefore, there is little conceptual value in considering organisms undertaking the first category to "co-direct their own evolution" or to generate adaptation.

The second category is considerably more exclusive. Processes that accelerate the adaptive evolution of the organisms undertaking them cause these organisms to adapt faster to ever-changing environments. These processes can be said not just to alter the organism's adaptations, but to increase the organism's adaptedness. Such processes can be shaped by natural selection for their evolutionary influence, in which case their generation of adaptations fits the criteria of autonomous selection.

The foregoing raises the notion that the category of developmental processes that most illustrate the inadequacy of the modern synthesis — those that most inspired the EES—overlaps fully with the category constituting autonomous selection. This can clarify the EES controversy substantially.

In recent reviews of the EES (Laland et al. 2014; Laland et al. 2015) and on a website devoted to the EES 5 , proponents have focused upon four phenomena in particular: 1) niche construction; 2) phenotypic plasticity; 3)

\footnotetext{
${ }^{5}$ extendedevolutionarysynthesis.com, last retrieved May 29, 2019
} 
developmental bias; and 4) nongenetic inheritance. Each of these four phenomena are defined extremely broadly, though EES proponents have narrowed the scope of these phenomena and focused upon subsets they consider particularly interesting and unaccommodated by the modern synthesis.

\subsection{Niche construction}

Niche construction (Odling-Smee et al. 2003, pg. 41) is "when an organism modifies the feature-factor relationship between itself and its environment by actively changing one or more of the factors in its environment, either by physically perturbing factors at its current location in space and time, or by relocating to a different space-time address, thereby exposing itself to different factors." Of this broad set of phenomena, Laland et al. (2014) emphasized the evolutionary effects upon an organism that result from those of the organism's actions that were shaped by selection. Contrasting them with "idiosyncratic events," they wrote that (pg. 162), for example, "termites construct and regulate their homes in a repeatable, directional manner that is shaped by past selection and that instigates future selection.” For Laland et al. (2014, pg. 162), when niche construction occurs in this manner, "organisms co-direct their own evolution by systematically changing environments and thereby biasing selection." Therefore, Laland (2004, pg. 316) encouraged a "re-conceptualization of the process of adaptation by placing emphasis on niche construction."

EES proponents have emphasized examples that involve organisms that systematically their environment, which alters natural selection upon the organisms. I refer to the discussion in section 7 and distinguish between two kinds of cases by which this occurs: 1) environment shaping that alters natural selection on the organisms shaping it as a byproduct of why the shaping occurs and 2) environment shaping that occurs for the purpose of favoring certain traits of the organisms shaping it. Only this second category constitutes autonomous selection. The first category violates criterion 3 .

The first category is illustrated by another example emphasized by EES proponents: The advent of dairy farming by humans led to selection for lactose tolerant alleles in farmers and other humans (Scott-Phillips et al. 2014). In this example, humans influenced their environment and, in the process, influenced selection upon themselves through their environment. However, humans did not conduct dairy farming for the purpose of favoring lactose tolerant alleles (or any others). The increase in frequency in lactose tolerant alleles is attributable to natural selection, not autonomous selection. 
The niche construction occurring in examples offered by EES proponents alters natural selection and, consequently, the adaptations that evolve, but so can any other change to the organism-environment relationship, including those resulting from random or quasirandom processes, such as genetic drift or mutation. What niche construction does not offer in these examples is the expectation of added adaptedness. This is the difference between natural selection and niche construction — a difference identifiable (though perhaps underappreciated) in the writings of EES proponents. Odling-Smee et al. (2003, pg. 18-19) wrote that: “(T)he argument that niche construction can be disregarded (as an evolutionary process) because it is partly a product of natural selection makes no more sense than would the counter proposal that natural selection can be disregarded because it is partly a product of niche construction. One cannot assume that the ultimate cause of niche construction is the environments that selected for niche-constructing traits, if prior niche construction had partly caused the state of the selective environments...Ultimately, such recursions would regress back to the beginning of life..." However, later in the same book, Odling-Smee et al. (2003, pg. 176) did acknowledge the unique role of natural selection in offering the expectation of adaptive evolution, writing that niche construction "must usually be restricted to fitness-enhancing behaviors or processes in the short term. Niche construction is unlikely to be random or haphazard because of prior natural selection."

In sum, niche construction can alter natural selection in such a way that different adaptations evolve in the niche-constructing population, but there is no reason for expecting these adaptations to be more adaptive than the adaptations that would have evolved in the absence of such niche construction. If there were such an expectation, then the niche construction could have been selected for this evolutionary influence. (The evolutionary influence would then constitute autonomous selection.)

\subsection{Phenotypic plasticity}

West-Eberhard defined (2003, pg. 33) phenotypic plasticity as "the ability of an organism to react to an internal or external environmental input with a change in form, state, movement, or rate of activity. It may or may not be adaptive (a consequence of previous selection)." Phenotypic plasticity is similar to niche construction in that it is a way to alter the organism-environment relationship — niche construction by altering the environment; phenotypic plasticity by altering the organism. Changing the organism-environment relationship alters the way natural selection occurs and influences which traits the organisms subsequently evolve, but it does not mean the plasticity has 
evolved for the purpose of favoring any traits. Furthermore, influencing the evolution of traits does not necessarily mean accelerating adaptive evolution.

It is worth noting that phenotypic plasticity is involved in numerous examples of autonomous selection that have been offered in this paper, including fitness-dependent sex, and horizontal gene transfer. However, I consider here specific examples of phenotypic plasticity raised by EES proponents.

In genetic assimilation (Waddington 1953), a phenotype that is initially expressed as a response to certain conditions becomes, after some generations of conditional exhibition of the phenotype, expressed even in the absence of the conditions that initially induced it. Laland et al. (2014) were referring to this effect of plasticity when they emphasized that "(P)lasticity not only allows organisms to cope in new environmental conditions but to generate traits that are well-suited to them...In other words, often it is the trait that comes first; genes that cement it follow, sometimes several generations later." EES proponents do not dispute that genetic assimilation occurs by natural selection, as random mutations (and random epigenetic changes) that make the traits more obligate offer fitness advantages. Genetic assimilation does not constitute an example of autonomous selection unless there is evidence that mutations are directed to making the traits more obligate.

Pigliucci $(2007,2010)$ has focused upon evolutionary capacitance. In evolutionary capacitance, there is an initial, "buffering" step, in which individuals accumulate different mutations and this genetic variation between the individuals is not associated with phenotypic variation because of the actions of regulatory processes that orient (i.e., buffer) the phenotype despite the genetic variation. Then there is a "revealing" step, in which some stimulant (e.g., a stressor) affects the regulatory processes in such a way that they no longer buffer the phenotype and, consequently, the genetic variation becomes associated with phenotypic variation. When there is phenotypic variation, natural selection can act, which often leads to genetic assimilation of phenotypic traits that are adaptive. This genetic assimilation, if it occurs, is the adaptive evolution that occurs.

Some biologists have argued that there can be natural selection of the buffering step because it prevents mutations from being immediately subjected to natural selection, which enables a beneficial combination of mutations to evolve when each mutation would be deleterious on its own (Kim 2007; Trotter et al. 2014). By my reading, this does not satisfy criterion 1: It has the effect of increasing the prevalence of individuals with mutations and, eventually, the prevalence of individuals with combinations of mutations, but it does not cause any allele to be 
more likely to be subject to mutation than any other. That is, the buffering increases the frequencies of mutations but does not exert a nonrandom influence upon which traits evolve. In these senses, it is similar to hypermutation (section 4).

Natural selection for a variant that does the revealing — counteracting the usual buffering and yielding phenotypic variation - would not constitute an example of autonomous selection, since the revealing itself does not constitute adaptive evolution (or evolution at all). However, if a case were made that natural selection shaped a variant to orient mutations in the direction of the revealed traits, that would be autonomous selection.

\subsection{Developmental bias}

Like niche construction and phenotypic plasticity, developmental bias has been defined to cover a very broad set of phenomena. Arthur (2004, pg. 207) wrote, “Consider any particular type of creature whose developmental trajectory can change in a certain number of ways-let's say 100. Does the probability of generating variant trajectories (ultimately via mutation and reprogramming) vary across these 100 directions...? ...(E)quiprobability is only one of an almost infinite series of patterns. All the others represent, by definition, various types and degrees of developmental bias." Like niche construction, developmental bias has been considered by some to have a focal role in the evolution of adaptations. For example, Arthur (2004, pg. 201) wrote, "Natural selection is not the 'main' orienting agent of evolution as Darwin claimed. Rather, it is one partner in an interacting duo that is the main determinant of the direction of evolutionary change...The other partner is developmental bias..."

There are numerous examples of developmental bias in which the underrepresented or unrepresented variants are thought to offer equal or higher adaptive value compared to the variants that are better represented but such variants are not as readily obtainable by random processes of mutation and recombination (Maynard Smith et al. 1985; Uller et al. 2018). Since the organism (and its lineage) is not expected to fit better to its environment as a result of such a bias, these examples of developmental bias do not result from natural selection shaping the bias itself. These cases illustrate that even though some biologists might put developmental bias on par with natural selection in directing adaptive evolution, natural selection differs from developmental bias in that evolution by natural selection can be expected to improve the fit of a target to its environment, whereas developmental bias does not necessarily. 
However, there are other examples of developmental bias in which the underrepresented or unrepresented variants are thought to offer lower adaptive value compared to the better represented variants. These are examples in which individuals (and their lineages) are better adapted to their environment than if the bias had not occurred. Some biologists have argued that natural selection can shape developmental bias (Parter et al. 2008; Pavlicev et al. 2011; Jones et al. 2014; Watson et al. 2014; Watson and Szathmary 2016; reviewed in Uller et al. 2018). A number of these authors have invoked the theory of facilitated variation, which proposes that organisms have properties that allow them to generate adaptive, heritable phenotypic variation (Kirschner and Gerhart 2005; Gerhart and Kirschner 2007; Kirschner and Gerhart 2010). Natural selection can shape pleiotropy and epistasis that cause mutations to change traits in combinations that are more likely to be adaptive than other combinations of changes (Uller et al. 2018). For example, there may be natural selection of pleiotropy and epistasis that cause mutational effects that make a right arm longer to cooccur with those that make a left arm longer. This causes organisms to be less likely to have arms of different lengths. In this manner, developmental bias can accelerate adaptive evolution because organisms do not waste evolutionary time by exhibiting maladaptive combinations of traits (Watson and Szathmary 2016; Uller et al. 2018).

Criterion 1: Is there nonrandom change in the frequency of one or more traits?

As a consequence of the pleiotropy and epistatic effects that have been discussed in examples of developmental bias, the frequency of maladaptive trait combinations is lower than otherwise. For example, developmental bias that causes mutational effects on left arm length to correlate with mutational effects on right arm length reduces the frequency of different arm lengths (presumably a maladaptive combination) in the same organism.

Criterion 2: Is the change sufficiently heritable to constitute evolution?

Developmental bias helps organisms adapt faster to environmental changes. It even allows organisms to adapt faster to environments that have the same structural regularity as prior environments but that are different in some ways from all previous environments (Uller et al. 2018). Developmental bias facilitates adaptation to these new environments with novel phenotypic combinations (Parter et al. 2008; Watson et al. 2014; Uller et al. 2018).

Criterion 3: Has natural selection shaped a variant to favor or disfavor one or more heritable traits? 
Biologists have demonstrated that natural selection can shape pleiotropy and epistasis that aligns mutational effects with the direction of the fitness landscape (reviewed in Uller et al. 2018). In models that demonstrate how natural selection shapes pleiotropy and epistasis to cause developmental bias, the genetic elements responsible for the pleiotropy and epistasis allow the organisms that hold these elements to be better-adapted to their environment than if they were to exhibit the maladaptive trait combinations that are prevented by the pleiotropy and epistasis. In these examples, there is natural selection of the pleiotropy and epistasis. Their prevention of maladaptive trait genetic combinations is autonomous selection against those combinations.

\subsection{Nongenetic inheritance}

EES proponents have emphasized that parents do not just pass genes to offspring, they also pass egg components, hormones, maternal care, and their environmental modifications (Laland et al. 2015). They argue that these factors can be a source of novel variation on which natural selection can operate. More pertinently to this manuscript, EES proponents argue that these modes of inheritance provide opportunities for acquired characters to be inherited. In section 7, I discuss some evidence for the inheritance of acquired adaptive characters. Additionally, under the "inclusive inheritance" section of their website, EES proponents mention the example of the evolution of lactose tolerance, which I have considered in the niche construction section (section 7.1).

\section{Conclusion}

The examples of autonomous selection examined in this manuscript involve variants shaped by natural selection to act upon the organisms in which they are located in a manner that lowers the frequency of traits associated with lesser adaptiveness and, in doing so, raises the frequency of traits associated with higher adaptiveness. As a consequence of this autonomous selection, the variants accelerate the evolution of adaptations of the organisms in which they are located.

In some examples, this acceleration of adaptive evolution results from stress-induced changes to genes and genetic combinations (e.g., stress-induced mutagenesis, section 3; fitness-dependent sex, section 5; horizontal genetic transfer, section 6.2): Genes and genetic combinations associated with lesser adaptiveness are continuously changed until genes and genetic combinations are generated that are associated with better adaptiveness. Acceleration of adaptive evolution also results from developmental bias (section 7.3) that biases mutational effects 
toward adaptiveness. And acceleration of adaptive evolution results from modifications that prevent a cell from becoming compromised (e.g., CRISPR, section 6.1; piRNA defenses, section 6.3; virophage defenses, section 6.4).

The environment constantly changes due to the evolution of predators, parasites, and prey and changes in weather. It generally takes time for adaptations in an environment to evolve and by the time they have evolved to an environment, the environment will have changed—in particular, by the evolution of natural enemies such as predators and parasites. Accelerating the evolution of adaptations lowers the time-lag and, therefore, improves the organisms' adaptive fit. The examples of autonomous selection examined in this manuscript all accelerate adaptive evolution and also cause organisms to be better-adapted than they would be if natural selection were acting in the absence of the variants responsible.

In reviews of the EES, proponents have not yet recognized a distinction between two kinds of processes: 1) processes that accelerate the adaptive evolution of the organisms responsible and 2) processes that merely alter the adaptive evolution of those organisms. As I have argued in this section, processes of the former kind cause the organisms to be better-adapted to their environment, while processes of the latter kind allow no such expectation. All of the examples of autonomous selection considered in this manuscript are processes of the former kind. Part of the utility of the concept, autonomous selection, is that it allows all known processes that increase the organism's adaptedness in its environment to be grouped under selection.

Many of the examples raised by EES proponents fit into the latter category. Processes of the latter category are joined in altering the adaptive evolution of the organisms responsible by many other processes, including the evolution of natural enemies and weather processes.

The foregoing is not an exhaustive review of examples of autonomous selection. Empirical advances are likely to inform whether other phenomena belong. For example, paramutation involves a nonrandom heritable change in trait frequency (Chandler 2007; Pilu 2011): A paramutable allele is induced to adopt the epigenetic state of a paramutagenic allele. However, biologists continue to investigate the purpose for which natural selection shaped paramutation, if it has (Charlesworth et al. 2017). Numerous biologists view paramutation to have adaptive significance for the organism (or some level higher) (see Jablonka and Lamb 2014, pgs. 493-494).

\section{References}


Agrawal AF (2006) Evolution of sex: why do organisms shuffle their genotypes? Curr Biol 16(17): 696-704

Al Mamun AAM, Lombardo MJ, Shee C, Lisewski AM, Gonzalez C, Lin D, Nehring RB, Saint-Ruf C, Gibson JL, Frisch RL, Lichtarge O, Hastings PJ, Rosenberg SM (2012) Identity and function of a large gene network underlying mutagenic repair of DNA breaks. Science 338(6112): 1344-1348

Aravin AA, Hannon GJ, Brennecke J (2007) The Piwi-piRNA pathway provides an adaptive defense in the transposon arms race. Science 318(5851): 761-764

Arthur W (2004) Biased Embryos and Evolution. Cambridge University Press, Cambridge, MA

Bell G (1997) The Basics of Selection. Chapman and Hall, New York

Brooker RJ, Widmaier EP, Graham LE, Stilling PD (2014) Biology, $3^{\text {rd }}$ edition. McGraw-Hill, New York

Burrus V, Waldor MK (2004) Shaping bacterial genomes with integrative and conjugative elements. Res. Microbiol. 155(5): $376-386$

Chandler VL (2007) Paramutation: from maize to mice. Cell 128(4): 641-645

Charlesworth D, Barton NH, Charlesworth B (2017) The sources of adaptive variation. Proc R Soc Lond B Biol Sci 284(1855): 20162864

Colegrave N (2012) The evolutionary success of sex. EMBO Reports 13(9): 774-778

Darwin C (1859) The Origin of Species by Means of Natural Selection. John Murray, London

Darwin C (1871) The Descent of Man and Selection in Relation to Sex. John Murray, London

Dickins TE, Barton RA (2012) Reciprocal causation and the proximate-ultimate distinction. Biol Philos 28(5): 747756

Dickins TE, Rahman Q (2012) The extended evolutionary synthesis and the role of soft inheritance in evolution. Proc R Soc Lond B Biol Sci 279(1740): 2913-2921

Domenech A, Slager J, Veening JW (2018) Antibiotic-induced cell chaining triggers pneumococcal competence by reshaping quorum sensing to autocrine-like signaling. Cell Rep 25(9): 2390-2400 
Fischer MG, Hackl T (2016) Host genome integration and giant virus-induced reactivation of the virophage mavirus. Nature 540(7632): 288-291

Futuyma DJ (2009) Evolution, $2^{\text {nd }}$ edition. Sinauer Associates, Sunderland, MA

Galhardo RS, Hastings PJ, Rosenberg SM (2007) Mutation as a stress response and the regulation of evolvability. Crit Rev Biochem Mol Biol 42(5): 399-435

Gerhart JC, Kirschner MW (2007) The theory of facilitated variation. Proc Natl Acad Sci USA 104(Suppl 1): 85828589

Gogarten JP, Doolittle WF, Lawrence JG (2002) Prokaryotic evolution in light of gene transfer. Mol Biol Evol 19(12): 2226-2238

Gogarten JP, Townsend JP (2005) Horizontal gene transfer, genome innovation and evolution. Nat Rev Microbiol 3(9): 679-687

Gross L (2006) Transposon silencing keeps jumping genes in their place. Plos Biol 4(10): e353

Gyles C, Boerlin P (2014) Horizontally transferred genetic elements and their role in pathogenesis of bacterial disease. Vet Pathol 51(2): 328-340

Hadany L, Beker T (2003) On the evolutionary advantage of fitness-associated recombination. Genetics 165(4): $2167-2179$

Hadany L, Beker T (2007) Sexual selection and the evolution of obligatory sex. BMC Evol Biol 7(1): 245

Hadany L, Otto SP (2007) The evolution of condition-dependent sex in the face of high costs. Genetics 176(3): $1713-1727$

Hadany L, Otto SP (2009) Condition-dependent sex and the rate of adaptation. Am Nat 174: S71-S78

Haig D (2013) Proximate and ultimate causes: How come? And what for? Biol Philos 28(5): 781-786

Halic M, Moazed D (2009) Transposon silencing by piRNAs. Cell 138(6): 1058-1060 
Harris JR (2006) Parental selection: a third selection process in the evolution of human hairlessness and skin color. Med. Hypotheses 66(6): 1053-1059

Hille F, Richter H, Wong SP, Bratovic M, Ressel S, Charpentier, E. (2018) The biology of CRISPR-Cas: backward and forward. Cell 172(6): 1239-1259

Hodgkinson A, Eyre-Walker A (2011) Variation in the mutation rate across mammalian genomes. Nat Rev Genet 12(11): 756-766

Horvath P, Barrangou R (2010) CRISPR/Cas, the immune system of bacteria and archaea. Science 327(5962): 167 170

Jablonka E, Lamb MJ (2005) Evolution in Four Dimensions. The MIT Press, Cambridge, MA.

Jablonka E, Lamb MJ (2008) Soft inheritance: challenging the modern synthesis. Genet Mol Biol 31(2): 389-395

Jablonka E, Lamb MJ (2010) Transgenerational epigenetic inheritance. In Pigliucci M, Muller GB (eds) Evolution: The Extended Synthesis. The MIT Press, Cambridge, MA, pgs. 137-174

Jablonka E, Lamb MJ (2014) Evolution in Four Dimensions, revised edition. The MIT Press, Cambridge, MA.

Jones AG, Burger R, Arnold SJ (2014) Epistasis and natural selection shape the mutational architecture of complex traits. Nat Commun 5: 3709

Kay E, Vogel TM, Bertolla F, Nalin R, Simonet P (2002) In situ transfer of antibiotic resistance genes from transgenic (transplastomic) tobacco plants to bacteria. Appl Environ Microbiol 68(7): 3345-3351

Keeling PJ, Palmer JD (2008) Horizontal gene transfer in eukaryotic evolution. Nat Rev Genet 9(8): 605-618

Kim Y (2007) Rate of adaptive peak shifts with partial genetic robustness. Evolution 61(8): 1847-1856

Kirschner MW, Gerhart JC (2005) The Plausibility of Life: Resolving Darwin’s Dilemma. Yale University Press, New Haven, CT

Kirschner MW, Gerhart JC (2010) Facilitated variation. In Pigliucci M, Muller GB (eds) Evolution: The Extended Synthesis. The MIT Press, Cambridge, MA, pgs. 253-280 
Koonin EV (2018) Open questions: CRISPR biology. BMC Biology 16: 95

Koonin EV (2019) CRISPR: a new principle of genome engineering linked to conceptual shifts in evolutionary biology. Biol Philos 34(1): 9

Koonin EV, Krupovic M (2016) Virology: a parasite's parasite saves host's neighbours. Nature 540(7632): 204-205

Koonin EV, Makarova KS, Aravind L (2001) Horizontal gene transfer in prokaryotes: quantification and classification. Annu Rev Microbiol 55: 709-742

Koonin EV, Wolf YI (2009) Is evolution Darwinian or/and Lamarckian? Biol Direct 4: 42

Koonin EV, Wolf YI (2016) Just how Lamarckian is CRISPR-Cas immunity: the continuum of evolvability mechanisms. Biol. Direct, 11: 9

Laland K (2004) Extending the extended phenotype. Biol Philos 19(3): 313-325

Laland KN, Uller T, Feldman MW, Sterelny K, Muller GB, Moczek A, Jablonka E, Odling-Smee J (2015) The extended evolutionary synthesis: its structure, assumptions and predictions. Proc R Soc Lond B Biol Sci 282(1813): 20151019

Laland KN, Uller T, Feldman MW, Sterelny K, Muller GB, Moczek A, Jablonka E, Odling-Smee J, Wray GA, Hoekstra HE, Futuyma DJ, Lenski RE, Mackay TFC, Schluter D, Strassmann JE (2014) Does evolutionary theory need a rethink? Nature 514(7521): 161-164

Lorch PD, Proulx S, Rowe L, Day T (2003) Condition-dependent sexual selection can accelerate adaptation. Evol Ecol Res 5: 867-881

MacLean RC, Torres-Barcelo C, Moxon R (2013) Evaluating evolutionary models of stress-induced mutagenesis. Nat Rev Genet 14(3): 221-227

Martin WF (2018) Eukaryote lateral gene transfer is Lamarckian. Nat Ecol Evol 2(5): 754

Martincorena I, Luscombe NM (2012) Non-random mutation: the evolution of targeted hypermutation and hypomutation. BioEssays 35(2) 123-130 
Martincorena I, Seshasayee ASN, Luscombe NM (2012) Evidence of non-random mutation rates suggests an evolutionary risk management strategy. Nature 485(7396): 95-98

Maynard Smith JM, Burian R, Kauffman SA, Alberch P, Campbell JH, Goodwin B, Lande R, Raup DM, Wolpert, L 1985. Developmental constraints and evolution: a perspective from the Mountain Lake Conference on development and evolution. Q Rev Biol 60(3): 265-287

Mayr E (1997) The objects of selection. Proc Natl Acad Sci USA 94(6): 2091-2094

Michod RE (2011) Evolutionary transitions in individuality: multicellularity and sex. In Calcott B, Sterelny K (eds) The Major Transitions in Evolution Revisited. The MIT Press, Cambridge, MA, pgs. 169-197

Mittelman D (ed) (2013) Stress-Induced Mutagenesis. Springer, New York

Nielsen KM, Bohn T, Townsend, JP (2014) Detecting rare gene transfer events in bacteria populations. Front Microbiol 4: 415

Ochman H, Lawrence JG, Groisman EA (2000) Lateral gene transfer and the nature of bacterial innovation. Nature 405(6784): 299-304

Odling-Smee FJ, Laland KN, Feldman M (2003) Niche Construction: The Neglected Process in Evolution. Monographs in Population Biology, 37. Princeton University Press, Princeton

O’Malley MA, Boucher Y (2005) Paradigm change in microbiology. Stud Hist Philos Biol Biomed Sci 36(1): 183208

Otto SP (2009) The evolutionary enigma of sex. Am Nat 174(Suppl 1): S1-S14

Parhad SS, Theurkauf WE (2019) Rapid evolution and conserved function of the piRNA pathway. Open Biol 9(1): 180181

Parter M, Kashtan N, Alon U (2008) Facilitated variation: how evolution learns from past environments to generalize to new environments. Plos Comput Biol 4(11): e1000206

Pavlicev M, Cheverud JM, Wagner GP (2011) Evolution of adaptive phenotypic variation patterns by direct selection for evolvability. Proc R Soc Lond B Biol Sci 278(1713): 1903-1912 
Pigliucci M (2007) Do we need an extended evolutionary synthesis? Evolution 61(12): 2743-2749

Pigliucci M (2010) Phenotypic plasticity. In Pigliucci M, Muller GB (eds) Evolution: The Extended Synthesis. The MIT Press, Cambridge, MA, pgs. 355-378

Pilu R (2011) Paramutation: just a curiosity or fine tuning of gene expression in the next generation? Curr Genomics 12(4): $298-306$

Polz MF, Alm EJ, Hanage WP (2013) Horizontal gene transfer and the evolution of bacterial and archaeal population structure. Trends Genet 29(3): 170-175

Ponder RG, Fonville NC, Rosenberg SM (2005) A switch from high-fidelity to error-prone DNA double-strand break repair underlies stress-induced mutation. Mol Cell 19(6): 791-804

Prudhomme M, Attaiech L, Sanchez G, Martin B, Claverys JP (2006) Antibiotic stress induces genetic transformability in the human pathogen/streptococcus pneumoniae. Science 313(5783): 89-92

Ram Y, Hadany L (2016) Condition-dependent sex: who does it, when and why? Philos Trans R Soc Lond B Biol Sci 371(1706): 20150539

Roger AJ (2018) Reply to “Eukaryote lateral gene transfer is Lamarckian.” Nat Ecol Evol 2(5): 755

Scott-Phillips TC, Laland KN, Shuker DM, Dickins TE, West SA (2014) The niche construction perspective: a critical appraisal. Evolution 68(5): 1231-1243

Shapiro JA (2011) Evolution: A View From the $21^{\text {st }}$ century. FT Press Science: Upper Saddle River, NJ

Shee C, Gibson JL, Darrow MC, Gonzalez C, Rosenberg SM (2011) Impact of a stress-inducible switch to mutagenic repair of DNA breaks on mutation in Escherichia coli. Proc Natl Acad Sci USA 108(33): 1365913664.

Siomi H, Siomi MC (2015) Phased piRNAs tackle transposons. Science 348(6236): 756-757

Slager J, Kjos M, Attaiech L, Veening JW (2014) Antibiotic-induced replication stress triggers bacterial competence by increasing gene dosage near the origin. Cell 157(2): 395-406 
Sorek R, Lawrence CM, Wiedenheft B (2013) CRISPR-mediated adaptive immune systems in bacteria and archaea. Annu Rev Biochem 82: 237-266

Soucy SM, Huang J, Gogarten JP (2015) Horizontal gene transfer: building the web of life. Nat Rev Genet 16(8): $472-482$

Stevens KE, Chang D, Zwack EE, Sebert ME (2011) Competence in Streptococcus pneumoniae is regulated by the rate of ribosomal decoding errors. mBio 2(5): e00071-11

Toth KF, Pezic D, Stuwe E, Webster A (2016) The piRNA pathway guards the germline genome against transposable elements. Adv Exp Med Biol 886: 51-77

Treangen TJ, Rocha EPC (2011) Horizontal transfer, not duplication, drives the expansion of protein families in prokaryotes. Plos Genet 7(1): e1001284

Trivers R, Burt A (2006) Genes in Conflict. Belknap: Cambridge, MA

Trotter MV, Weissman DB, Peterson GI, Peck KM, Masel J (2014) Cryptic genetic variation can make "irreducible complexity" a common mode of adaptation in sexual populations. Evolution 68(12): 3357-3367

Uller T, Moczek AP, Watson RA, Brakefield PM, Laland KN (2018) Developmental bias and evolution: a regulatory network perspective. Genetics 209(4): 949-966

Vetsigian K, Woese C, Goldenfeld N (2006) Collective evolution and the genetic code. Proc Natl Acad Sci USA 103(28): 10696-10701

Vogan AA, Higgs PG (2011) The advantages and disadvantages of horizontal gene transfer and the emergence of the first species. Biol Direct 6: 1

Vos M, Hesselman MC, Te Beek TA, van Passel MWJ, Eyre-Walker A (2015) Rates of lateral gene transfer in prokaryotes: high but why? Trends Microbiol 23(10): 598-605

Waddington CH (1953) Genetic assimilation of an acquired character. Evolution 7(2): 118-126

Watson RA, Szathmary E (2016) How can evolution learn? Trends Ecol Evol 31(2): 147-157 
Watson RA, Wagner GP, Pavlicev M, Weinreich DM, Mills R (2014. The evolution of phenotypic correlations and “developmental memory.” Evolution 68: 1124-1138

Weiss A (2015) Lamarckian illusions. Trends Ecol Evol 30(10): 566-568

West-Eberhard MJ (2003) Developmental Plasticity and Evolution. Oxford University Press: Oxford

Wilson EO (2014) The Meaning of Human Existence. Liveright Publishing Corporation: New York

Zirkle C (1935) The inheritance of acquired characters and the provisional hypothesis of pangenesis. Am Nat 69(72): 417-445 